OPEN ACCESS

Edited by:

Ravid Doron,

Open University of Israel, Israel

Reviewed by: Juan Francisco Rodríguez-Landa, Universidad Veracruzana, Mexico Keren Nitzan,

Open University of Israel, Israel

*Correspondence:

Paul J. Fitzgerald pfitz1940@gmail.com

Specialty section: This article was submitted to Emotion Regulation and Processing, a section of the journal Frontiers in Behavioral Neuroscience

Received: 28 February 2021 Accepted: 07 September 2021 Published: 30 September 2021

Citation:

Fitzgerald PJ (2021) Are Noradrenergic Transmission Reducing Drugs Antidepressants? Front. Behav. Neurosci. 15:673634 doi: 10.3389/fnbeh.2021.673634

\section{Are Noradrenergic Transmission Reducing Drugs Antidepressants?}

\author{
Paul J. Fitzgerald* \\ Department of Psychiatry, University of Michigan, Ann Arbor, MI, United States
}

Major depressive disorder (MDD) remains a significant public health problem worldwide, and revised treatment strategies are therefore urgently needed, including the creation of novel antidepressant compounds or using existing molecular entities in new ways. Etiologic theories of MDD from decades ago have suggested that synaptic deficiencies of monoaminergic neurotransmitters play a causative role in this neuropsychiatric disorder, and that boosting monoamines with drugs such as SSRIs, SNRIs, TCAs, and MAOls has antidepressant effects and in some individuals can even induce hypomania or mania. While other factors, such as various intracellular molecular pathways and hippocampal neurogenesis, undoubtedly also play a role in MDD, monoaminergic boosting drugs nonetheless have clearly demonstrated antidepressant properties. There is also, however, a body of studies in the preclinical literature suggesting that monoaminergic transmission reducing drugs, including noradrenergic ones, also have antidepressant-like behavioral properties in rodents. Given that there is increasing evidence that the monoamines have u-shaped or Janus-faced dose-response properties, in which a mid-range value is "optimal" in a variety of behavioral and physiological processes, it is plausible that either too much or too little synaptic norepinephrine in key circuits may exacerbate MDD in some individuals. Here we briefly review rodent depression-related behavioral data, focusing on the forced swim test, from three major classes of noradrenergic transmission reducing drugs (alpha2 agonists, beta blockers, alpha1 antagonists), and find much support for the hypothesis that they have antidepressant-like properties. Whether these drugs are antidepressants in human subjects remains to be determined.

\section{Keywords: norepinephrine, noradrenaline, serotonin, SSRI (selective serotonergic reuptake inhibitors),} propranolol, clonidine, guanfacine, prazosin

\section{INTRODUCTION}

Despite intensive efforts by commercial and academic researchers for many decades, major depressive disorder (MDD) remains a significant source of morbidity and mortality throughout the world (Chen et al., 2017; Schmaal et al., 2017; Hasin et al., 2018; Ingram et al., 2020). Many individuals who experience MDD do not respond completely, or in some cases at all, to existing pharmacological or behavioral treatment modalities, leaving a need for new approaches (Mitchell, 2004; Ruhé et al., 2006; Ulrich et al., 2020). 
In addition to the demand for improved psychotherapeutic treatments, the field would benefit from the creation of novel pharmacological agents or the repurposing of existing compounds that may, perhaps unexpectedly, have beneficial properties in the treatment of MDD (Ebada, 2017; Demin et al., 2019).

Following the discovery of some of today's widely used antidepressants (MAOIs, TCAs) in the mid-20th century, monoaminergic theories on the etiology of MDD were put forth, suggesting that diminished brain levels of serotonin, norepinephrine (NE), and dopamine are a causative factor in the disorder (Schildkbaut, 1965; Coppen, 1967; Janowsky et al., 1972). In the decades since then, it has become increasingly clear that a number of intracellular molecular pathways (which undoubtedly interact with the extracellular monoamines) also play a role in MDD and the physiological and behavioral responses to antidepressant drugs (Vaidya and Duman, 2001; Tanis and Duman, 2007; Miller et al., 2009; Wohleb et al., 2016), although the continued medical use of antidepressants that boost synaptic monoamines (including SSRIs, SNRIs, NDRIs, TCAs, MAOIs) reinforces the clinical utility of this approach.

For these reasons, it may be surprising to note that in the preclinical literature there is also a significant body of studies suggesting that noradrenergic transmission reducing drugs, such as the alpha 2 agonist clonidine, exhibit antidepressant-like behavioral properties under a variety of experimental conditions. This may be a surprising finding since a number of the monoaminergic theories of MDD suggest that elevated monoamines should produce mania or hypomania (Schildkbaut, 1965; Coppen, 1967; Janowsky et al., 1972), and by inference transmission reducing drugs may have mood-stabilizing properties but not necessarily be antidepressants. However, a growing body of evidence suggests that endogenous serotonin, NE, and dopamine have u-shaped or Janus-faced dose-response properties for a range of behaviors, wherein too much or too little signaling may be pathological (Baldi and Bucherelli, 2005; Arnsten, 2007; Vijayraghavan et al., 2007; Giustino et al., 2016; Giustino and Maren, 2018; Groft et al., 2019). In this scenario, perhaps a non-optimal (i.e., decreased or elevated) synaptic concentration of each monoamine may result in MDD, at least in some individuals with the disorder.

Below we briefly review rodent preclinical findings on the depression-related behavioral effects of three major classes of noradrenergic transmission reducing drugs: alpha2 agonists, beta blockers, and alphal antagonists. We focus on three major behavioral assays: the forced swim test (FST), the tail suspension test (TST), and the sucrose preference test. We conducted a PubMed database search using the following terms (February 7 , 2021): clonidine/guanfacine/dexmedetomidine/propranolol/ carvedilol/nebivolol/metoprolol/atenolol/prazosin/"beta

blocker(s)"/alpha1/alpha2/beta1/beta2/beta3 +"forced swim" /"forced swimming"/"tail suspension"/"sucrose preference" /antidepressant-like/depression-like. This literature search yielded a total of 489 publications. Forty-eight were judged to be relevant articles that included data with at least one of the above types of drugs (alpha2 agonists, beta blockers, alpha1 antagonists), in mice or rats that were exposed to at least one of the above three behavioral assays (FST, TST, sucrose preference). To be included, these papers had to be published in the English language, and the 48 that met these criteria are further described in Table 1. There was no limit set on how long ago the papers were published. We did not focus on studies that investigated the interaction between natural products or compounds and these noradrenergic agents.

\section{ALPHA2 AGONISTS}

Dating back several decades, there is a body of evidence suggesting that alpha2 adrenergic agonists such as clonidine and guanfacine, which inhibit the presynaptic release of $\mathrm{NE}$ and activate alpha 2 receptors that are also located postsynaptically, have antidepressant-like properties in rodent models. While there are some opposing data suggesting that alpha2 antagonists can have antidepressant-like effects (Muguruza et al., 2013; Uys et al., 2017), a number of studies report that alpha2 agonists such as clonidine are therapeutic when administered acutely. A number of these studies indeed suggest that clonidine, by itself, can produce antidepressant-like effects in tests such as the FST (Malinge et al., 1988, 1989; Cervo and Samanin, 1991; Cervo et al., 1992; Asakura et al., 1993, 1994; Skrebuhhova et al., 1999; Masuda et al., 2001; O’Neill et al., 2001; Malikowska et al., 2017).

Clonidine, in many cases, when given at sub-effective doses, can also potentiate the antidepressant-like effects of a wide range of other drugs that have antidepressant properties such as SSRIs, NDRIs, TCAs, MAOIs, 5HT1A agonists, lithium, lamotrigine, and others (Malinge et al., 1988, 1989; Bourin et al., 1991, 1996, 2002; Hascoät et al., 1991; Hascoet et al., 1994; Redrobe and Bourin, 1997, 1998; Skrebuhhova et al., 1999; Kaster et al., 2007; Zeidan et al., 2007; Taksande et al., 2009; Kotagale et al., 2013). In some cases these effects were shown to be counteracted by alpha2 antagonists such as idazoxan or yohimbine, suggesting clonidine achieves its antidepressant-like properties through activation of the alpha2 receptor (Malinge et al., 1988, 1989; Cervo and Samanin, 1991; Masuda et al., 2001; O’Neill et al., 2001; Zeidan et al., 2007).

In contrast to these potentially therapeutic properties of clonidine, it has also been suggested that this drug can promote depression-like behavior in rodents (Kitada et al., 1983; Parale and Kulkarni, 1986; Ferrari et al., 1991; Rénéric et al., 2002), or under some circumstances has no substantial effect either alone or when co-administered with other putative antidepressants (Kitada et al., 1983; Evangelista et al., 1987; Antkiewicz-Michaluk et al., 2017).

It has also been shown that molecular overexpression of alpha2C adrenoceptors can decrease immobility in the mouse FST (Sallinen et al., 1999), perhaps mimicking the antidepressant-like effects of alpha2 agonists such as clonidine. Antidepressant-like effects of two other alpha2 agonists, guanfacine and dexmedetomidine, have also been reported in rodent models (Stone et al., 2011; Mineur et al., 2015, 2018). 
TABLE 1 | Summary of antidepressant-related effects of noradrenergic transmission reducing drugs.

\begin{tabular}{|c|c|c|c|c|c|c|c|c|c|c|c|c|}
\hline Publication & Species & Strain & Sex & Primary Drug & $\begin{array}{l}\text { Secondary } \\
\text { Drug }\end{array}$ & $\begin{array}{l}\text { Dose } \\
\text { (mg/kg) }\end{array}$ & Route & Repeats & $\begin{array}{l}\text { Time } \\
\text { Delay }\end{array}$ & Stress & Test & Effect \\
\hline \multirow[t]{3}{*}{ O'Neill et al. (2001) } & M & BKTO & $\mathrm{F}$ & clonidine & none & 0.25 & s.c. & 0 & $30 \mathrm{~min}$ & none & FS & dec imm \\
\hline & & & & clonidine & none & 0.5 & s.c. & 0 & $30 \mathrm{~min}$ & none & FS & dec imm \\
\hline & & & & clonidine & none & 1 & s.c. & 0 & $30 \mathrm{~min}$ & none & FS & dec imm \\
\hline \multirow{2}{*}{$\begin{array}{l}\text { Malikowska et al. } \\
\text { (2017) }\end{array}$} & M & CD-1 & M & clonidine & none & 0.1 & i.p. & 0 & $60 \mathrm{~min}$ & none & FS & dec imm \\
\hline & M & $C D-1$ & M & clonidine & none & 0.1 & i.p. & 0 & $60 \mathrm{~min}$ & $24 \mathrm{~h}$ after & FS & n.s. \\
\hline \multirow{6}{*}{$\begin{array}{l}\text { Asakura et al. } \\
\text { (1993) }\end{array}$} & M & $d d Y$ & M & clonidine & none & 0.03 & i.p. & 0 & $30 \mathrm{~min}$ & none & FS & n.s. \\
\hline & & & & clonidine & none & 0.1 & i.p. & 0 & $30 \mathrm{~min}$ & none & FS & n.s. \\
\hline & & & & clonidine & none & 0.3 & i.p. & 0 & $30 \mathrm{~min}$ & none & FS & inc swim \\
\hline & & & & clonidine & none & 0.03 & i.p. & 0 & $30 \mathrm{~min}$ & 48 h soc & FS & n.s. \\
\hline & & & & clonidine & none & 0.1 & i.p. & 0 & $30 \mathrm{~min}$ & 48 h soc & FS & n.s. \\
\hline & & & & clonidine & none & 0.3 & i.p. & 0 & $30 \mathrm{~min}$ & 48 h soc & FS & inc swim \\
\hline \multirow{8}{*}{$\begin{array}{l}\text { Asakura et al. } \\
\text { (1994) }\end{array}$} & M & $d d Y$ & M & clonidine & none & 0.03 & i.p. & 0 & $30 \mathrm{~min}$ & none & FS & n.s. \\
\hline & & & & clonidine & none & 0.1 & i.p. & 0 & $30 \mathrm{~min}$ & none & FS & n.s. \\
\hline & & & & clonidine & none & 0.3 & i.p. & 0 & $30 \mathrm{~min}$ & none & FS & inc swim \\
\hline & & & & clonidine & none & 1 & i.p. & 0 & $30 \mathrm{~min}$ & none & FS & inc swim \\
\hline & & & & clonidine & none & 0.03 & i.p. & 0 & $30 \mathrm{~min}$ & $48 \mathrm{~h} \mathrm{so}$ & FS & n.s. \\
\hline & & & & clonidine & none & 0.1 & i.p. & 0 & $30 \mathrm{~min}$ & 48 h soc & FS & n.s. \\
\hline & & & & clonidine & none & 0.3 & i.p. & 0 & $30 \mathrm{~min}$ & 48 h soc & FS & inc swim \\
\hline & & & & clonidine & none & 1 & i.p. & 0 & $30 \mathrm{~min}$ & 48 h soc & FS & inc swim \\
\hline \multirow{3}{*}{$\begin{array}{l}\text { Masuda et al. } \\
\text { (2001) }\end{array}$} & M & $d d Y$ & M & clonidine & none & 0.004 & i.p. & 0 & $45 \mathrm{~min}$ & none & FS & inc clim \\
\hline & & & & clonidine & none & 0.02 & i.p. & 0 & $45 \mathrm{~min}$ & none & FS & inc clim \\
\hline & & & & clonidine & none & 0.1 & i.p. & 0 & $45 \mathrm{~min}$ & none & FS & inc clim \\
\hline Kaster et al. (2007) & M & Swiss & $\mathrm{F}$ & clonidine & none & 0.06 & i.p. & 0 & $60 \mathrm{~min}$ & none & FS & n.s. \\
\hline \multirow{2}{*}{$\begin{array}{l}\text { Kotagale et al. } \\
\text { (2013) }\end{array}$} & M & Swiss & M & clonidine & none & 0.015 & i.p. & 0 & $30 \mathrm{~min}$ & none & FS & n.s. \\
\hline & & & & clonidine & bupropion & $\begin{array}{l}0.015 \\
5\end{array}$ & i.p. & 0 & $30 \mathrm{~min}$ & none & FS & dec imm \\
\hline \multirow{4}{*}{$\begin{array}{l}\text { Hascoät et al. } \\
\text { (1991) }\end{array}$} & M & Swiss & M & clonidine & none & 0.06 & i.p. & 0 & $30 \mathrm{~min}$ & none & FS & n.s. \\
\hline & & & & clonidine & none & 0.125 & i.p. & 0 & $30 \mathrm{~min}$ & none & FS & inc mob \\
\hline & & & & clonidine & none & 0.25 & i.p. & 0 & $30 \mathrm{~min}$ & none & FS & n.s. \\
\hline & & & & clonidine & none & 0.5 & i.p. & 0 & $30 \mathrm{~min}$ & none & FS & inc mob \\
\hline
\end{tabular}


TABLE 1 | Continued

\begin{tabular}{|c|c|c|c|c|c|c|c|c|c|c|c|c|}
\hline Publication & Species & Strain & Sex & Primary Drug & $\begin{array}{l}\text { Secondary } \\
\text { Drug }\end{array}$ & $\begin{array}{l}\text { Dose } \\
\text { (mg/kg) }\end{array}$ & Route & Repeats & $\begin{array}{l}\text { Time } \\
\text { Delay }\end{array}$ & Stress & Test & Effect \\
\hline & & & & clonidine & none & 1 & i.p. & 0 & $30 \mathrm{~min}$ & none & FS & inc mob \\
\hline & & & & clonidine & none & 0.06 & i.p. & 0 & $30 \mathrm{~min}$ & none & TS & n.s. \\
\hline & & & & clonidine & none & 0.125 & i.p. & 0 & $30 \mathrm{~min}$ & none & TS & n.s. \\
\hline & & & & clonidine & none & 0.25 & i.p. & 0 & $30 \mathrm{~min}$ & none & TS & dec imm \\
\hline & & & & clonidine & none & 0.5 & i.p. & 0 & $30 \mathrm{~min}$ & none & TS & n.s. \\
\hline & & & & clonidine & none & 1 & i.p. & 0 & $30 \mathrm{~min}$ & none & TS & n.s. \\
\hline \multirow{16}{*}{$\begin{array}{l}\text { Malinge et al. } \\
\text { (1988) }\end{array}$} & M & Swiss & M & clonidine & none & 0.015 & i.p. & 0 & $30 \mathrm{~min}$ & none & FS & n.s. \\
\hline & & & & clonidine & none & 0.06 & i.p. & 0 & $30 \mathrm{~min}$ & none & FS & inc mob \\
\hline & & & & clonidine & none & 0.25 & i.p. & 0 & $30 \mathrm{~min}$ & none & FS & inc mob \\
\hline & & & & clonidine & none & 1 & i.p. & 0 & $30 \mathrm{~min}$ & none & FS & inc mob \\
\hline & & & & clonidine & none & 4 & i.p. & 0 & $30 \mathrm{~min}$ & none & FS & inc mob \\
\hline & & & & clonidine & none & 16 & i.p. & 0 & $30 \mathrm{~min}$ & none & FS & inc mob \\
\hline & & & & clonidine & imipramine & $0.06,4$ & i.p. & 0 & $30 \mathrm{~min}$ & none & FS & dec imm \\
\hline & & & & clonidine & amitriptyline & $0.06,1$ & i.p. & 0 & $30 \mathrm{~min}$ & none & FS & dec imm \\
\hline & & & & clonidine & maprotiline & $0.06,8$ & i.p. & 0 & $30 \mathrm{~min}$ & none & FS & dec imm \\
\hline & & & & clonidine & mianserin & $0.06,4$ & i.p. & 0 & $30 \mathrm{~min}$ & none & FS & dec imm \\
\hline & & & & clonidine & viloxazine & $0.06,2$ & i.p. & 0 & $30 \mathrm{~min}$ & none & FS & dec imm \\
\hline & & & & clonidine & citalopram & $0.06,2$ & i.p. & 0 & $30 \mathrm{~min}$ & none & FS & dec imm \\
\hline & & & & clonidine & indalpine & $0.06,4$ & i.p. & 0 & $30 \mathrm{~min}$ & none & FS & dec imm \\
\hline & & & & clonidine & fluvoxamine & $0.06,4$ & i.p. & 0 & $30 \mathrm{~min}$ & none & FS & dec imm \\
\hline & & & & clonidine & inprindole & $\begin{array}{l}0.06, \\
32\end{array}$ & i.p. & 0 & $30 \mathrm{~min}$ & none & FS & dec imm \\
\hline & & & & clonidine & nialamide & $\begin{array}{l}0.06 \\
32\end{array}$ & i.p. & 0 & $30 \mathrm{~min}$ & none & FS & dec imm \\
\hline \multirow[t]{6}{*}{ Bourin et al. (1991) } & M & Swiss & M & clonidine & imipramine & $0.1,8$ & i.p. & 0 & $30 \mathrm{~min}$ & none & FS & dec imm \\
\hline & & & & clonidine & amitriptyline & $0.1,2$ & i.p. & 0 & $30 \mathrm{~min}$ & none & FS & dec imm \\
\hline & & & & clonidine & maprotiline & $0.1,8$ & i.p. & 0 & $30 \mathrm{~min}$ & none & FS & dec imm \\
\hline & & & & clonidine & citalopram & $0.1,4$ & i.p. & 0 & $30 \mathrm{~min}$ & none & FS & dec imm \\
\hline & & & & clonidine & fluvoxamine & $0.1,8$ & i.p. & 0 & $30 \mathrm{~min}$ & none & FS & dec imm \\
\hline & & & & clonidine & paroxetine & $0.1,8$ & i.p. & 0 & $30 \mathrm{~min}$ & none & FS & dec imm \\
\hline \multirow[t]{5}{*}{ Bourin et al. (1996) } & M & Swiss & M & clonidine & imipramine & $0.06,4$ & i.p. & 0 & $30 \mathrm{~min}$ & none & FS & dec imm \\
\hline & & & & clonidine & fluoxetine & $0.06,2$ & i.p. & 0 & $30 \mathrm{~min}$ & none & FS & dec imm \\
\hline & & & & clonidine & trazodone & $\begin{array}{l}0.06 \\
0.5\end{array}$ & i.p. & 0 & $30 \mathrm{~min}$ & none & FS & dec imm \\
\hline & & & & clonidine & mianserin & $0.06,4$ & i.p. & 0 & $30 \mathrm{~min}$ & none & FS & dec imm \\
\hline & & & & clonidine & gepirone & $0.06,4$ & i.p. & 0 & $30 \mathrm{~min}$ & none & FS & dec imm \\
\hline \multirow[t]{2}{*}{ Bourin et al. (2002) } & M & Swiss & M & clonidine & tranylcypromine & $\begin{array}{l}0.06 \\
0.5\end{array}$ & i.p. & 0 & $30 \mathrm{~min}$ & none & FS & dec imm \\
\hline & & & & clonidine & phenelzine & $0.06,8$ & i.p. & 0 & $30 \mathrm{~min}$ & none & FS & dec imm \\
\hline
\end{tabular}

(Continued) 
TABLE 1 | Continued

\begin{tabular}{|c|c|c|c|c|c|c|c|c|c|c|c|c|}
\hline Publication & Species & Strain & Sex & Primary Drug & $\begin{array}{l}\text { Secondary } \\
\text { Drug }\end{array}$ & $\begin{array}{l}\text { Dose } \\
(\mathrm{mg} / \mathrm{kg})\end{array}$ & Route & Repeats & $\begin{array}{l}\text { Time } \\
\text { Delay }\end{array}$ & Stress & Test & Effect \\
\hline \multirow{3}{*}{$\begin{array}{l}\text { Hascoet et al. } \\
\text { (1994) }\end{array}$} & M & Swiss & M & clonidine & 8-OH-DPAT & $0.1,0.5$ & i.p. & 0 & $30 \mathrm{~min}$ & none & FS & n.s. \\
\hline & & & & clonidine & gepirone & $0.1,4$ & i.p. & 0 & $30 \mathrm{~min}$ & none & FS & dec imm \\
\hline & & & & clonidine & ipsapirone & $0.1,1$ & i.p. & 0 & $30 \mathrm{~min}$ & none & FS & dec imm \\
\hline \multirow{7}{*}{$\begin{array}{l}\text { Redrobe and } \\
\text { Bourin (1997) }\end{array}$} & M & Swiss & M & clonidine & imipramine & $0.06,4$ & i.p. & 0 & $30 \mathrm{~min}$ & none & TS & dec imm \\
\hline & & & & clonidine & fluoxetine & $0.06,2$ & i.p. & 0 & $30 \mathrm{~min}$ & none & TS & dec imm \\
\hline & & & & clonidine & trazodone & $\begin{array}{l}0.06 \\
0.5\end{array}$ & i.p. & 0 & $30 \mathrm{~min}$ & none & TS & dec imm \\
\hline & & & & clonidine & mianserin & $0.06,4$ & i.p. & 0 & $30 \mathrm{~min}$ & none & TS & dec imm \\
\hline & & & & clonidine & iprindole & $\begin{array}{l}0.06 \\
32\end{array}$ & i.p. & 0 & $30 \mathrm{~min}$ & none & TS & n.s. \\
\hline & & & & clonidine & ritanserin & $\begin{array}{l}0.06 \\
0.5\end{array}$ & i.p. & 0 & $30 \mathrm{~min}$ & none & TS & dec imm \\
\hline & & & & clonidine & ipsapirone & $0.06,1$ & i.p. & 0 & $30 \mathrm{~min}$ & none & TS & dec imm \\
\hline \multirow{3}{*}{$\begin{array}{l}\text { Redrobe and } \\
\text { Bourin (1998) }\end{array}$} & M & Swiss & M & clonidine & 8-OH-DPAT & $0.06,1$ & i.p. & 0 & $30 \min$ & none & FS & dec imm \\
\hline & & & & clonidine & ritanserin & $0.06,4$ & i.p. & 0 & $30 \mathrm{~min}$ & none & FS & dec imm \\
\hline & & & & clonidine & ketanserin & $0.06,8$ & i.p. & 0 & $30 \mathrm{~min}$ & none & FS & n.s. \\
\hline \multirow{6}{*}{$\begin{array}{l}\text { Taksande et al. } \\
\text { (2009) }\end{array}$} & M & Swiss & M & clonidine & none & 0.015 & i.p. & 0 & $30 \mathrm{~min}$ & none & FS & n.s. \\
\hline & & & & clonidine & none & 0.03 & i.p. & 0 & $30 \mathrm{~min}$ & none & FS & n.s. \\
\hline & & & & clonidine & none & 0.06 & i.p. & 0 & $30 \mathrm{~min}$ & none & FS & dec imm \\
\hline & & & & clonidine & imipramine & $\begin{array}{l}0.015 \\
2.5\end{array}$ & i.p. & 0 & $30 \mathrm{~min}$ & none & FS & n.s. \\
\hline & & & & clonidine & fluoxetine & $\begin{array}{l}0.015 \\
2.5\end{array}$ & i.p. & 0 & $30 \mathrm{~min}$ & none & FS & dec imm \\
\hline & & & & clonidine & paroxetine & $\begin{array}{l}0.015 \\
2.5\end{array}$ & i.p. & 0 & $30 \mathrm{~min}$ & none & FS & dec imm \\
\hline \multirow[t]{2}{*}{ Zeidan et al. (2007) } & M & Swiss & $\begin{array}{l}M, \\
F\end{array}$ & clonidine & none & 0.06 & i.p. & 0 & $30 \mathrm{~min}$ & none & FS & n.s. \\
\hline & & & & clonidine & agmatine & $\begin{array}{l}0.06 \\
0.001\end{array}$ & i.p. & 0 & $30 \mathrm{~min}$ & none & FS & dec imm \\
\hline \multirow[t]{2}{*}{ Ferrari et al. (1991) } & M & Swiss & M & clonidine & none & 0.075 & i.p. & 0 & $25 \min$ & none & TS & inc imm \\
\hline & & & & clonidine & none & 0.15 & i.p. & 0 & $25 \mathrm{~min}$ & none & TS & inc imm \\
\hline \multirow{3}{*}{$\begin{array}{l}\text { Parale and Kulkarni } \\
\text { (1986) }\end{array}$} & M & Wist & M & clonidine & none & 0.05 & i.p. & 0 & $15 \mathrm{~min}$ & none & FS & inc imm \\
\hline & & & & clonidine & none & 0.15 & i.p. & 0 & $15 \mathrm{~min}$ & none & FS & inc imm \\
\hline & & & & clonidine & none & 0.5 & i.p. & 0 & $15 \mathrm{~min}$ & none & FS & inc imm \\
\hline $\begin{array}{l}\text { Evangelista et al. } \\
\text { (1987) }\end{array}$ & $\mathrm{R}$ & CD-COBS & M & clonidine & none & 0.1 & i.p. & 0 & $30 \mathrm{~min}$ & none & FS & n.s. \\
\hline \multirow{4}{*}{$\begin{array}{l}\text { Cervo and Samanin } \\
\text { (1991) }\end{array}$} & $\mathrm{R}$ & S-D & M & clonidine & none & 0.05 & i.p. & 0 & $30 \mathrm{~min}$ & none & FS & n.s. \\
\hline & & & & clonidine & none & 0.1 & i.p. & 0 & $30 \mathrm{~min}$ & none & FS & n.s. \\
\hline & & & & clonidine & none & 0.5 & i.p. & 0 & $30 \mathrm{~min}$ & none & FS & n.s. \\
\hline & & & & clonidine & none & 0.05 & i.p. & 2 & $30 \mathrm{~min}$ & none & FS & dec imm \\
\hline
\end{tabular}


TABLE 1 | Continued

\begin{tabular}{|c|c|c|c|c|c|c|c|c|c|c|c|c|}
\hline Publication & Species & Strain & Sex & Primary Drug & $\begin{array}{l}\text { Secondary } \\
\text { Drug }\end{array}$ & $\begin{array}{l}\text { Dose } \\
\text { (mg/kg) }\end{array}$ & Route & Repeats & $\begin{array}{l}\text { Time } \\
\text { Delay }\end{array}$ & Stress & Test & Effect \\
\hline & & & & clonidine & none & 0.1 & i.p. & 2 & $30 \mathrm{~min}$ & none & FS & dec imm \\
\hline & & & & clonidine & none & 0.5 & i.p. & 2 & $30 \mathrm{~min}$ & none & FS & dec imm \\
\hline & & & & clonidine & none & 0.1 & i.p. & $\begin{array}{l}\text { b.i.d. for } \\
15 \text { days }\end{array}$ & $30 \mathrm{~min}$ & none & FS & n.s. \\
\hline Cervo et al. (1992) & $\mathrm{R}$ & S-D & M & clonidine & none & 0.1 & i.p. & 2 & $30 \mathrm{~min}$ & none & FS & dec imm \\
\hline \multirow[t]{2}{*}{ Kitada et al. (1983) } & $\mathrm{R}$ & S-D & M & clonidine & none & 0.3 & s.c. & 2 & $30 \mathrm{~min}$ & none & FS & n.s. \\
\hline & & & & clonidine & desipramine & $0.3,20$ & s.c., i.p. & 2 & $30 \mathrm{~min}$ & none & FS & inc imm \\
\hline \multirow[t]{4}{*}{ Rénéric et al. (2002) } & $\mathrm{R}$ & S-D & M & clonidine & none & 0.005 & i.p. & 2 & $60 \mathrm{~min}$ & none & FS & inc swim \\
\hline & & & & clonidine & none & 0.01 & i.p. & 2 & $60 \mathrm{~min}$ & none & FS & n.s. \\
\hline & & & & clonidine & none & 0.02 & i.p. & 2 & $60 \mathrm{~min}$ & none & FS & n.s. \\
\hline & & & & clonidine & none & 0.2 & i.p. & 2 & $60 \mathrm{~min}$ & none & FS & inc clim \\
\hline \multirow{3}{*}{$\begin{array}{l}\text { Skrebuhhova et al. } \\
\text { (1999) }\end{array}$} & $\mathrm{R}$ & Wist & M & clonidine & none & 0.1 & i.p. & 1 & $30 \mathrm{~min}$ & none & FS & n.s. \\
\hline & & & & clonidine & none & 1 & i.p. & 1 & $30 \mathrm{~min}$ & none & FS & dec imm \\
\hline & & & & clonidine & desipramine & $0.1,10$ & i.p. & 1 & $15 \mathrm{~min}$ & none & FS & dec imm \\
\hline $\begin{array}{l}\text { Antkiewicz- } \\
\text { Michaluk et al. } \\
\text { (2017) }\end{array}$ & $\mathrm{R}$ & Wist & M & clonidine & none & 0.1 & i.p & 0 & $60 \mathrm{~min}$ & none & FS & inc clim \\
\hline \multirow[t]{8}{*}{ Mineur et al. (2015) } & M & C57 & $\begin{array}{l}\mathrm{M}, \\
\mathrm{F}\end{array}$ & guanfacine & none & 0.05 & i.p. & 0 & $30 \mathrm{~min}$ & none & FS & n.s. \\
\hline & & & & guanfacine & none & 0.1 & i.p. & 0 & $30 \mathrm{~min}$ & none & FS & n.s. \\
\hline & & & & guanfacine & none & 0.15 & i.p. & 0 & $30 \mathrm{~min}$ & none & FS & dec imm \\
\hline & & & & guanfacine & none & 0.3 & i.p. & 0 & $30 \mathrm{~min}$ & none & FS & n.s. \\
\hline & & & & guanfacine & none & 0.05 & i.p. & $\begin{array}{l}\text { q.d. for } \\
15 \text { days }\end{array}$ & $\begin{array}{l}\text { approx } \\
24 \mathrm{~h}\end{array}$ & none & FS & n.s. \\
\hline & & & & guanfacine & none & 0.1 & i.p. & $\begin{array}{l}\text { q.d. for } \\
15 \text { days }\end{array}$ & $\begin{array}{l}\text { approx } \\
24 \mathrm{~h}\end{array}$ & none & FS & dec imm \\
\hline & & & & guanfacine & none & 0.15 & i.p. & $\begin{array}{l}\text { q.d. for } \\
15 \text { days }\end{array}$ & $\begin{array}{l}\text { approx } \\
24 \mathrm{~h}\end{array}$ & none & FS & dec imm \\
\hline & & & & guanfacine & none & 0.3 & i.p. & $\begin{array}{l}\text { q.d. for } \\
15 \text { days }\end{array}$ & $\begin{array}{l}\text { approx } \\
24 \mathrm{~h}\end{array}$ & none & FS & n.s. \\
\hline \multirow[t]{2}{*}{ Mineur et al. (2018) } & M & C57 & $\begin{array}{l}\mathrm{M}, \\
\mathrm{F}\end{array}$ & guanfacine & none & 0.15 & i.p. & 0 & $30 \mathrm{~min}$ & none & FS & dec imm \\
\hline & & & & guanfacine & none & 0.15 & i.p. & 0 & $30 \mathrm{~min}$ & none & TS & dec imm \\
\hline $\begin{array}{l}\text { Parale and Kulkarni } \\
\text { (1986) }\end{array}$ & M & Wist & M & guanfacine & none & 0.15 & i.p. & 0 & $15 \mathrm{~min}$ & none & FS & inc imm \\
\hline \multirow[t]{2}{*}{ Stone et al. (2011) } & M & S-W & M & dexmedetomidine & none & $0.04 \mathrm{nmol}$ & i.c.v. & 0 & $5 \mathrm{~min}$ & none & TS & dec imm \\
\hline & & & & dexmedetomidine & none & $0.1 \mathrm{nmol}$ & i.c.v. & 0 & $5 \min$ & none & TS & dec imm \\
\hline \multirow{2}{*}{$\begin{array}{l}\text { Al-Tubuly et al. } \\
\text { (2008) }\end{array}$} & M & albino & NS & propranolol & none & 1 & i.p. & 0 & $60 \mathrm{~min}$ & none & FS & $\begin{array}{l}\text { dec latency } \\
\text { to imm }\end{array}$ \\
\hline & & & & propranolol & imipramine & 1,10 & i.p. & 0 & $60 \mathrm{~min}$ & none & FS & $\begin{array}{l}\text { dec latency } \\
\text { to imm }\end{array}$ \\
\hline
\end{tabular}




\begin{tabular}{|c|c|c|c|c|c|c|c|c|c|c|c|c|}
\hline Publication & Species & Strain & Sex & Primary Drug & $\begin{array}{l}\text { Secondary } \\
\text { Drug }\end{array}$ & $\begin{array}{l}\text { Dose } \\
\text { (mg/kg) }\end{array}$ & Route & Repeats & $\begin{array}{l}\text { Time } \\
\text { Delay }\end{array}$ & Stress & Test & Effect \\
\hline \multirow[t]{2}{*}{$\begin{array}{l}\text { Sekio and Seki } \\
\text { (2015) }\end{array}$} & M & CD-1 & M & propranolol & LPS & $\begin{array}{l}5 \mu \mathrm{l} \\
400 \mathrm{mM}\end{array}$ & i.c.v. & 0 & $24 \mathrm{~h}$ & none & FS & n.s. \\
\hline & & & & propranolol & LPS & $\begin{array}{l}5 \mu \mathrm{l} \\
400 \mathrm{mM}\end{array}$ & i.c.v. & 0 & $24 \mathrm{~h}$ & none & TS & n.s. \\
\hline \multirow[t]{2}{*}{ Zhang et al. (2009) } & M & FVB & M & propranolol & none & 2.5 & i.p. & 0 & $45 \mathrm{~min}$ & none & FS & n.s. \\
\hline & & & & propranolol & desipramine & $2.5,20$ & i.p. & 0 & $30 \mathrm{~min}$ & none & FS & n.s. \\
\hline Gu et al. (2012) & M & ICR & M & propranolol & none & 5 & i.p. & 0 & $120 \mathrm{~min}$ & none & TS & n.s. \\
\hline \multirow[t]{4}{*}{ Teste et al. (1990) } & M & NMRI & M & propranolol & none & 0.12 & i.p. & 0 & $60 \mathrm{~min}$ & none & TS & n.s. \\
\hline & & & & propranolol & none & 0.5 & i.p. & 0 & $60 \mathrm{~min}$ & none & TS & n.s. \\
\hline & & & & propranolol & none & 2 & i.p. & 0 & $60 \mathrm{~min}$ & none & TS & n.s. \\
\hline & & & & propranolol & none & 8 & i.p. & 0 & $60 \mathrm{~min}$ & none & TS & n.s. \\
\hline $\begin{array}{l}\text { Pesarico et al. } \\
\text { (2014) }\end{array}$ & M & Swiss & M & propranolol & none & 2 & i.p. & 0 & $45 \mathrm{~min}$ & none & FS & n.s. \\
\hline $\begin{array}{l}\text { Evangelista et al. } \\
\text { (1987) }\end{array}$ & $\mathrm{R}$ & CD-COBS & M & propranolol & none & 5 & i.p. & 0 & $120 \mathrm{~min}$ & none & FS & n.s. \\
\hline \multirow{6}{*}{$\begin{array}{l}\text { Abel and Hannigan } \\
\text { (1994) }\end{array}$} & $\mathrm{R}$ & F344 & M & propranolol & none & 1 & i.p. & 0 & $60 \mathrm{~min}$ & none & FS & n.s. \\
\hline & & & & propranolol & none & 3 & i.p. & 0 & $60 \mathrm{~min}$ & none & FS & inc imm \\
\hline & & & & propranolol & none & 5 & i.p. & 0 & $60 \mathrm{~min}$ & none & FS & inc imm \\
\hline & & & & propranolol & none & 1 & i.p. & $\begin{array}{l}\text { q.d. for } \\
10 \text { days }\end{array}$ & $60 \mathrm{~min}$ & none & FS & n.s. \\
\hline & & & & propranolol & none & 3 & i.p. & $\begin{array}{l}\text { q.d. for } \\
10 \text { days }\end{array}$ & $60 \mathrm{~min}$ & none & FS & n.s. \\
\hline & & & & propranolol & none & 5 & i.p. & $\begin{array}{l}\text { q.d. for } \\
10 \text { days }\end{array}$ & $60 \mathrm{~min}$ & none & FS & n.s. \\
\hline $\begin{array}{l}\text { Finnegan et al. } \\
\text { (1987) }\end{array}$ & R & S-D & M & propranolol & none & 5 & i.p. & $\begin{array}{l}\text { q.d. for } \\
7 \text { days }\end{array}$ & $24 \mathrm{~h}$ & none & FS & n.s. \\
\hline \multirow[t]{2}{*}{ Zaidi et al. (2020) } & $\mathrm{R}$ & S-D & M & propranolol & none & 50/day & in water & $\begin{array}{l}\text { given for } \\
36 \text { days }\end{array}$ & 9 days & none & FS & n.s. \\
\hline & & & & propranolol & none & 50/day & in water & $\begin{array}{l}\text { given for } \\
36 \text { days }\end{array}$ & 9 days & soc defe & FS & dec imm \\
\hline Aisa et al. (2008) & $\mathrm{R}$ & Wist & $\mathrm{F}$ & propranolol & none & 2 & s.c. & 0 & $60 \mathrm{~min}$ & mat sep & FS & dec imm \\
\hline \multirow[t]{2}{*}{ Zaidi et al. (2020) } & $\mathrm{R}$ & S-D & M & nadolol & none & 18/day & in chow & $\begin{array}{l}\text { given for } \\
36 \text { days }\end{array}$ & 9 days & none & FS & n.s. \\
\hline & & & & nadolol & none & 18/day & in chow & $\begin{array}{l}\text { given for } \\
36 \text { days }\end{array}$ & 9 days & $\mathrm{soc} d$ & FS & dec imm \\
\hline Park et al. (2012) & M & C57 & M & butoxamine & none & 5 & i.p. & 1 & $30 \mathrm{~min}$ & none & FS & n.s. \\
\hline \multirow[t]{2}{*}{$\begin{array}{l}\text { Al-Tubuly et al. } \\
\text { (2008) }\end{array}$} & M & albino & NS & atenolol & none & 5 & i.p. & 0 & $60 \mathrm{~min}$ & none & FS & $\begin{array}{l}\text { inc latency } \\
\text { to imm }\end{array}$ \\
\hline & & & & atenolol & imipramine & 5,10 & i.p. & 0 & $60 \mathrm{~min}$ & none & FS & n.s. \\
\hline \multirow{2}{*}{$\begin{array}{l}\text { Stone and } \\
\text { Quartermain (1999) }\end{array}$} & M & S-W & M & betaxolol & none & 5 & i.p. & 0 & $20 \mathrm{~min}$ & none & TS & n.s. \\
\hline & & & & betaxolol & none & 20 & i.p. & 0 & $20 \mathrm{~min}$ & none & TS & n.s. \\
\hline \multirow[t]{2}{*}{ Detke et al. (1995) } & $\mathrm{R}$ & S-D & M & betaxolol & none & 10 & s.c. & 2 & $60 \mathrm{~min}$ & none & FS & n.s. \\
\hline & & & & betaxolol & 8-OH-DPAT & $10,0.5$ & s.c. & 2 & $60 \mathrm{~min}$ & none & FS & n.s. \\
\hline Zaidi et al. (2020) & $\mathrm{R}$ & S-D & M & bisoprolol & none & $15 /$ day & in water & $\begin{array}{l}\text { given for } \\
36 \text { days }\end{array}$ & 9 days & none & FS & n.s. \\
\hline
\end{tabular}




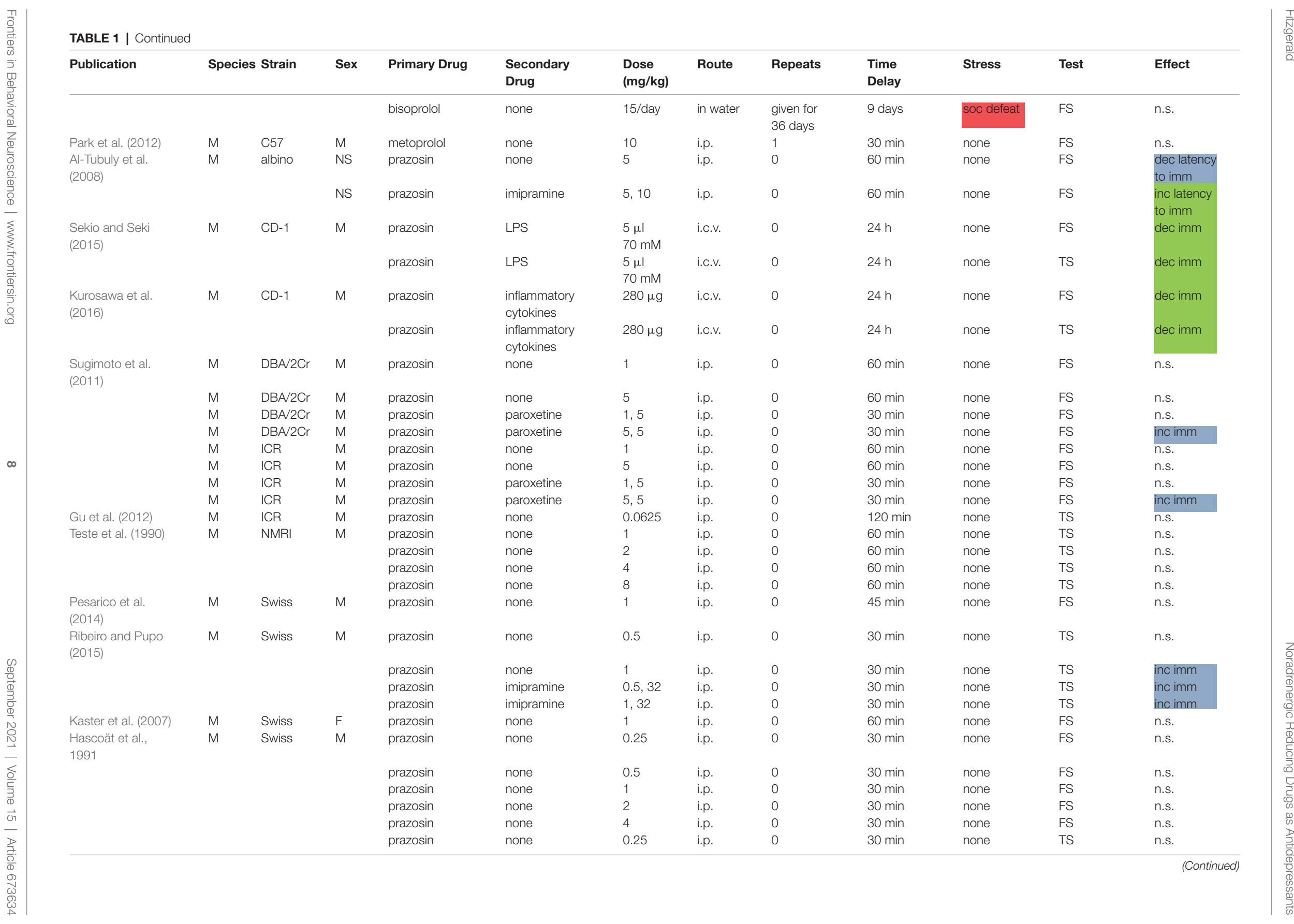


TABLE 1 | Continued

\begin{tabular}{|c|c|c|c|c|c|c|c|c|c|c|c|c|}
\hline Publication & Species & Strain & Sex & Primary Drug & $\begin{array}{l}\text { Secondary } \\
\text { Drug }\end{array}$ & $\begin{array}{l}\text { Dose } \\
\text { (mg/kg) }\end{array}$ & Route & Repeats & $\begin{array}{l}\text { Time } \\
\text { Delay }\end{array}$ & Stress & Test & Effect \\
\hline & & & & prazosin & none & 0.5 & i.p. & 0 & $30 \mathrm{~min}$ & none & TS & n.s. \\
\hline & & & & prazosin & none & 1 & i.p. & 0 & $30 \mathrm{~min}$ & none & TS & n.s. \\
\hline & & & & prazosin & none & 2 & i.p. & 0 & $30 \mathrm{~min}$ & none & TS & inc mob \\
\hline & & & & prazosin & none & 4 & i.p. & 0 & $30 \mathrm{~min}$ & none & TS & inc mob \\
\hline \multirow{2}{*}{$\begin{array}{l}\text { Stone and } \\
\text { Quartermain (1999) }\end{array}$} & M & S-W & M & prazosin & none & 0.5 & i.p. & 0 & $20 \mathrm{~min}$ & none & TS & inc imm \\
\hline & & & & prazosin & none & 2 & i.p. & 0 & $20 \mathrm{~min}$ & none & TS & inc imm \\
\hline $\begin{array}{l}\text { Evangelista et al. } \\
\text { (1987) }\end{array}$ & $\mathrm{R}$ & CD-COBS & M & prazosin & none & 3 & s.c. & 0 & $90 \mathrm{~min}$ & none & FS & n.s. \\
\hline $\begin{array}{l}\text { Poncelet et al. } \\
\text { (1987) }\end{array}$ & $\mathrm{R}$ & S-D & M & prazosin & desipramine & 2,32 & i.p. & 0 & $30 \mathrm{~min}$ & none & FS & inc imm \\
\hline $\begin{array}{l}\text { Cervo and Samanin } \\
\text { (1991) }\end{array}$ & $\mathrm{R}$ & S-D & M & prazosin & none & 3 & s.c. & 0 & $60 \mathrm{~min}$ & none & FS & n.s. \\
\hline \multirow[t]{2}{*}{ Detke et al. (1995) } & $\mathrm{R}$ & S-D & M & prazosin & none & 1 & s.c. & 2 & $60 \mathrm{~min}$ & none & FS & n.s. \\
\hline & & & & prazosin & 8-OH-DPAT & $1,0.5$ & s.c. & 2 & $60 \mathrm{~min}$ & none & FS & n.s. \\
\hline \multirow{4}{*}{$\begin{array}{l}\text { Schreiber and De } \\
\text { Vry (1993) }\end{array}$} & $\mathrm{R}$ & Wist & M & prazosin & none & 0.1 & i.p. & 2 & $60 \mathrm{~min}$ & none & FS & n.s. \\
\hline & & & & prazosin & none & 0.3 & i.p. & 2 & $60 \mathrm{~min}$ & none & FS & dec imm \\
\hline & & & & prazosin & 8-OH-DPAT & $0.1,3$ & i.p. & 2 & $60 \mathrm{~min}$ & none & FS & n.s. \\
\hline & & & & prazosin & 8-OH-DPAT & $0.3,3$ & i.p. & 2 & $60 \mathrm{~min}$ & none & FS & n.s. \\
\hline Stone et al. (2011) & M & S-W & M & terazosin & none & $1 \mathrm{nmol}$ & i.c.v. & 0 & $5 \mathrm{~min}$ & none & TS & inc imm \\
\hline \multirow[t]{2}{*}{ Wu et al. (2017) } & $\mathrm{R}$ & S-D & M & benoxathian & none & $5 \mu \mathrm{g}$ & $\begin{array}{l}\text { prelimbic } \\
\text { infusion }\end{array}$ & 0 & $10 \mathrm{~min}$ & none & FS & inc imm \\
\hline & & & & benoxathian & none & $5 \mu \mathrm{g}$ & $\begin{array}{l}\text { prelimbic } \\
\text { infusion }\end{array}$ & 0 & $10 \mathrm{~min}$ & none & $\mathrm{SP}$ & $\begin{array}{l}\text { dec sucr } \\
\text { pref }\end{array}$ \\
\hline
\end{tabular}

This table comprises mouse and rat studies from our literature search that used these drugs in the forced swim (FS), tail suspension (TS), or sucrose preference (SP) tests. The "Repeats" column indicates how many times a drug treatment was repeated in that group of animals, where zero repeats indicate a single administration of that drug or drug pair. The "Time Delay" column represents the amount of time between the last (or only) administration of the drug or pair of drugs and when the behavioral test was carried out. The "Stress" column indicates whether an acute or chronic stressor was administered prior to testing. The "Effect" column describes the type of statistically significant outcome in the behavioral test or otherwise shows that the result was not statistically significant (n.s.; $p>0.05$ ). For single drug administration, the Effect column describes the effect relative to vehicle administration. For pairs of drugs, the Effect column compares the behavioral effect of the pair with when one drug alone was given in that experiment. All experiments that used a prior stressor are marked in red. Experiments that showed a statistically significant antidepressant-like effect are marked in green, whereas those with a depression-like effect are marked in blue. Other abbreviations: [Species: mouse (M), rat (R)], C57BL/6J (C57), Sprague-Dawley (S-D), Swiss Webster (S-W), Wistar (Wist), [Sex: male (M), female (F)], lipopolysaccharide (LPS), intraperitoneal (i.p.), subcutaneous (s.c.), intracerebroventricular (i.c.v.), once a day (q.d.), twice a day (b.i.d.), social defeat (soc defeat), maternal separation (mat sep), social isolation (soc isol), single prolonged stress (SPS), decreased immobility (dec imm), increased immobility (inc imm), increased mobility (inc mob), increased swimming (inc swim), increased climbing (inc clim), decreased sucrose preference (dec sucr pref). 


\section{BETA BLOCKERS}

Beta blockers such as propranolol and nadolol (non-selective beta1/2 antagonists), metoprolol and atenolol (beta1), and butoxamine (beta2) can exhibit antidepressant-like activity in the FST (Chopra et al., 1988; Beǐer, 1994; Aisa et al., 2008; Park et al., 2012; Zaidi et al., 2020), including potentiation of sub-effective doses of other putative antidepressants such as baclofen (Aley and Kulkarni, 1990), or antagonizing depression-like effects of other agents (Parale et al., 1987). A mouse study of propranolol and nadolol found that whereas these two drugs did not exhibit therapeutic effects in the TST, propranolol did show an antidepressant-like decrease in TST-induced hyperthermia (Liu et al., 2003). The non-selective beta blocker nebivolol has been shown to counteract the depression-like behavioral and pathophysiological effects of the chemotherapeutic agent cisplatin (Abdelkader et al., 2017). An immunocytochemical study of propranolol showed that it could reduce the number of cells that stained for Fos-like immunoreactivity in various subcortical and cortical regions, resembling standard antidepressants such as imipramine and desipramine (Duncan et al., 1996).

In contrast to these potentially therapeutic properties of beta blockers, it has also been suggested that these drugs can promote depression-like behavior in rodents (Abel and Hannigan, 1994; Stone and Quartermain, 1999; Al-Tubuly et al., 2008) including in the presence of other putative antidepressants (Zhang et al., 2009; Gu et al., 2012), or under some circumstances, they have no substantial effect either alone or when co-administered with other putative antidepressants (Danysz et al., 1986; Evangelista et al., 1987; Finnegan et al., 1987; Teste et al., 1990; Beèr, 1994; Detke et al., 1995; Pesarico et al., 2014; Sekio and Seki, 2015; Zaidi et al., 2020). A number of studies also suggest that the beta3 agonist amibegron (also called SR58611A) has antidepressant-like properties in rodents (Consoli et al., 2007; Overstreet et al., 2008; Stemmelin et al., 2008, 2010; Tamburella et al., 2010), and it may achieve these effects by modulating serotonergic and noradrenergic signaling that is triggered by activation of beta3 receptors (Claustre et al., 2008).

\section{ALPHA1 ANTAGONISTS}

Alpha1 antagonists such as prazosin and benoxathian can also exhibit antidepressant-like activity in the FST or TST (Sekio and Seki, 2015; Kurosawa et al., 2016; Wu et al., 2017), including potentiation of other putative antidepressants such as imipramine (Al-Tubuly et al., 2008). In contrast, it has also been suggested that alphal antagonists can promote depression-like behavior (Stone and Quartermain, 1999; AlTubuly et al., 2008), including in the presence of other putative antidepressants or electroconvulsive therapy (ECT; Danysz et al., 1986; Poncelet et al., 1987; Teste et al., 1990; Kaster et al., 2007; Sugimoto et al., 2011; Gu et al., 2012; Ribeiro and Pupo, 2015). Under some circumstances they have no substantial depression-related behavioral effect either alone or when co-administered with other putative antidepressants (Evangelista et al., 1987; Malinge et al., 1988, 1989; Cervo and Samanin, 1991; Schreiber and De Vry, 1993; Detke et al., 1995; Sugimoto et al., 2011; Pesarico et al., 2014). In addition, mice expressing constitutively active mutant alpha1 A (but not alpha1B) adrenoceptors exhibit antidepressant-like activity in the FST and TST, that is counteracted by prazosin (Doze et al., 2009).

Table 1 summarizes the results from, and experimental parameters used in the above rodent studies on noradrenergic transmission reducing drugs in the FST, TST, and sucrose preference test. A brief analysis of the table suggests a few prominent themes or findings: (1) clonidine is the drug with the most experimental evidence supporting an antidepressant-like role. Those data support its therapeutic-like role across a variety of both mouse and rat strains, in both the FST and TST, and an amplifying beneficial role when paired with a wide range of established antidepressants; (2) there is less support at this time of an antidepressant-like role for various beta blockers and the alphal antagonist prazosin, where a number of studies show depression-like effects for these drugs (although other data are supportive). These drugs appear to not have been studied as extensively in these tests as clonidine; (3) very few of the studies used female mice, which should be a priority in future studies, especially considering that the rate of MDD in women is approximately twice that in men (Baxter et al., 2014; Albert, 2015); (4) only a few of the studies used C57BL/6J mice, which are widely used in behavioral neuroscience, and could be combined with studies of additional strains of mice in further investigations; and (5) prior exposure to chronic stress, which can induce MDD in susceptible human subjects (Hosang et al., 2014; Bonde et al., 2016), was rarely used in these studies and should be further addressed with additional experiments.

\section{DISCUSSION}

The preclinical data reviewed above address the issue of whether noradrenergic transmission reducing pharmacological agents have antidepressant-like behavioral properties in rodents. While many of these studies, perhaps most numerously and convincingly for the alpha2 agonist clonidine, suggest that these drugs have therapeutic effects, a number of the publications found no effect or depression-like effects, including for the beta blocker propranolol and the alphal antagonist prazosin. How do we reconcile such opposing effects across studies for these drugs? Some possibilities are that they may be due to genetic differences across strains or species of animals, varying responses to acute or chronic stress, or in some cases different behavioral tests that were used. Another explanation is that since there may be a u-shaped or Janusfaced dose-response relationship for noradrenergic signaling (Arnsten, 2007; Giustino et al., 2016; Giustino and Maren, 2018), the different drug doses used in the above studies could have opposing behavioral effects, including through interaction with divergent cortical and subcortical circuits, 
which may vary across species and strain. If alpha2 agonists such as clonidine and guanfacine really do have more robust antidepressant-like properties than beta blockers and alpha1 antagonists, this may relate to the more general effect of alpha2 agonists decreasing the presynaptic release of NE (Gresch et al., 1995; Van Gaalen et al., 1997), which would in principle affect signaling at all subtypes of adrenoceptors simultaneously.

A number of the studies reviewed above investigated the interaction of noradrenergic transmission reducing agents with other types of drugs. Several of these studies suggest that these noradrenergic drugs can potentiate the antidepressant-like effects of SSRIs or 5HT1A agonists (Malinge et al., 1988, 1989; Bourin et al., 1991, 1996; Hascoet et al., 1994; Redrobe and Bourin, 1997, 1998; Taksande et al., 2009), although not all studies or data were supportive (Redrobe and Bourin, 1998; Rénéric et al., 2002). Despite these discrepancies, this may be a treatment strategy that has clinical ramifications for the pharmacotherapy of MDD in human subjects. It has been previously suggested (Dremencov et al., 2007a,b; Guiard et al., 2008; Fitzgerald and Watson, 2019) that serotonin and NE may have functionally opposed properties, which is consistent with the hypothesis that noradrenergic transmission reducing drugs can amplify the effects of SSRIs under some conditions. We also suggest here, consistent with a statement in our prior publication (Polis et al., 2019), that noradrenergic transmission reducing drugs may be antidepressants in a subset of humans suffering from MDD, who would also be responsive to the rapidly acting antidepressant ketamine, and to ECT. In this scenario, noradrenergic transmission reducing agents may interact with glutamatergic signaling to chronically suppress neural hyperexcitability associated with some cases of MDD (Figure 1), and possibly have rapid therapeutic onset like ketamine (Polis et al., 2019). While the molecular mechanisms through which noradrenergic transmission reducing drugs may achieve antidepressant-like effects are not well understood at this time, one possibility is that they selectively dampen certain intracellular signaling pathways after acting upon alpha and beta-adrenergic $G$ protein-coupled receptors. There is already evidence, for example, that NE modulates the Ras/MAPK, PI3K/Akt, JAK/STAT pathways (Muthalif et al., 1998; Yanagawa et al., 2010; Guo et al., 2013; Maity et al., 2020).

One might argue that noradrenergic transmission reducing drugs are, based on monoaminergic theories of mood disorders, more likely to have mood-stabilizing than antidepressant properties. After all, beta blockers such as propranolol have historically been more associated with induction of MDD or depressive-like symptomatology (Koella, 1985; Rosen and Kostis, 1985) (but also see: Kim et al., 2019; Kessing et al., 2020), or with attenuation of hypomania or mania (Emrich et al., 1979; Nemeth and Mckenzie Chustz, 2020), where the latter property has also been attributed to clonidine (Hardy et al., 1986; Nemeth and Mckenzie Chustz, 2020). One possibility is that if these drugs really are antidepressants under some conditions, they achieve these effects in individuals who exhibit

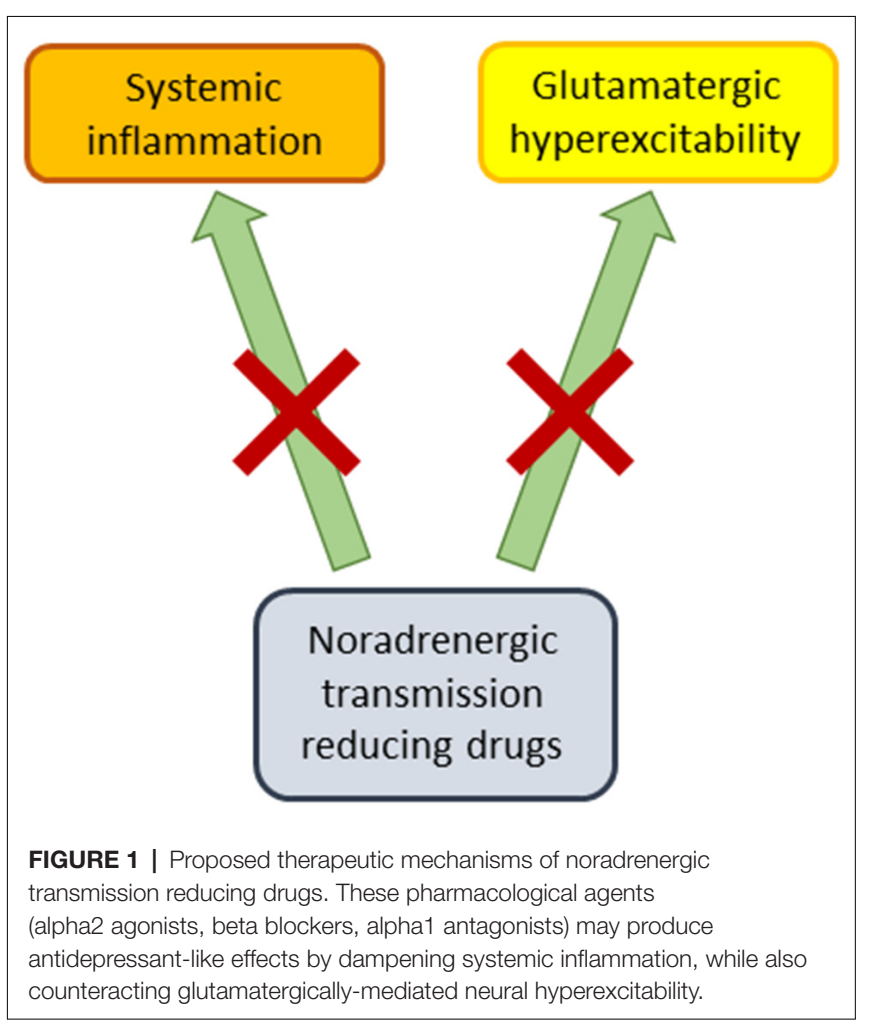

neural "decoupling" of NE with dopamine in mood-related circuits. In such an individual, elevated noradrenergic signaling may result in MDD rather than dopamine-facilitated hypomania or mania (Diehl and Gershon, 1992). Since MDD is also associated with systemic inflammation (Miller et al., 2009), noradrenergic transmission reducing agents may also produce antidepressant effects by counteracting neuroinflammation (Chen et al., 2015; Ding et al., 2019; Apple et al., 2020; Figure 1).

In conclusion, while there are conflicting data in rodents as to whether noradrenergic transmission reducing drugs have antidepressant-like properties, a number of studies reviewed above support this hypothesis, at least under some experimental conditions. At present, it is not clear whether neural noradrenergic transmission is elevated or suppressed in MDD (Waldmeier, 1981), where perhaps each state exists in different individuals. For these reasons, additional preclinical, mechanistic studies are needed, including those that induce depression-like behavior in animal models through the use of chronic mild stress. Based on the foundation of preclinical studies reviewed briefly here, further investigation of noradrenergic transmission reducing drugs in human mood disorders also appears warranted.

\section{AUTHOR CONTRIBUTIONS}

The author alone conceived of, researched, wrote, and edited this publication. 


\section{REFERENCES}

Abdelkader, N. F., Saad, M. A., and Abdelsalam, R. M. (2017). Neuroprotective effect of nebivolol against cisplatin-associated depressive-like behavior in rats. J. Neurochem. 141, 449-460. doi: 10.1111/jnc.13978

Abel, E. L., and Hannigan, J. H. (1994). The immobility response in the forced swim test: paradoxical effect of imipramine. Eur. J. Pharmacol. 258, 261-264. doi: 10.1016/0014-2999(94)90488-x

Aisa, B., Tordera, R., Lasheras, B., Del Río, J., and Ramírez, M. J. (2008). Effects of maternal separation on hypothalamic-pituitary-adrenal responses, cognition and vulnerability to stress in adult female rats. Neuroscience 154, 1218-1226. doi: 10.1016/j.neuroscience.2008.05.011

Albert, P. R. (2015). Why is depression more prevalent in women? J. Psychiatry Neurosci. 40, 219-221. doi: 10.1503/jpn.150205

Aley, K., and Kulkarni, S. (1990). Effect of baclofen, a GABAB-agonist, on forced swimming-induced immobility in mice. Arch. Int. Pharmacodyn. Ther. 307, $18-31$

Al-Tubuly, R. A., Aburawi, S. M., Alghzewi, E. A., Gorash, Z. M., and Errwami, S. (2008). The effect of sympathetic antagonists on the antidepressant action of alprazolam. Libyan J. Med. 3, 78-83. doi: 10.4176/080101

Antkiewicz-Michaluk, L., Romańska, I., Wঞsik, A., and Michaluk, J. (2017). Antidepressant-like effect of the endogenous neuroprotective amine, 1MeTIQ in clonidine-induced depression: behavioral and neurochemical studies in rats. Neurotox. Res. 32, 94-106. doi: 10.1007/s12640-0179715-z

Apple, C. G., Miller, E. S., Loftus, T. J., Kannan, K. B., Thompson, C. W., Lopez, M. C., et al. (2020). Effect of $\beta$-blockade on the expression of regulatory MicroRNA after severe trauma and chronic stress. J. Am. Coll. Surg. 230, 121-129. doi: 10.1016/j.jamcollsurg.2019.09.011

Arnsten, A. F. T. (2007). Catecholamine and second messenger influences on prefrontal cortical networks of "representational knowledge": a rational bridge between genetics and the symptoms of mental illness. Cereb. Cortex 17, i6-i15. doi: $10.1093 /$ cercor/bhm033

Asakura, W., Matsumoto, K., Ohta, H., Watanabe, H., Asakura, W., Matsumoto, K., et al. (1993). Effect of a 2-adrenergic drugs on REM sleep deprivation-induced increase in swimming activity. Pharmacol. Biochem. Behav. 46, 111-115. doi: 10.1016/0091-3057(93)90325-n

Asakura, W., Matsumoto, K., Ohta, H., Watanabe, H., Matsumoto, K., Ohta, H., et al. (1994). Monoamine depletion attenuates the rem sleep deprivationinduced increase in clonidine response in the forced swimming test. Pharmacol. Biochem. Behav. 49, 79-84. doi: 10.1016/0091-3057(94)90459-6

Baldi, E., and Bucherelli, C. (2005). The inverted "u-shaped" dose-effect relationships in learning and memory: modulation of arousal and consolidation. Nonlinearity Biol. Toxicol. Med. 3, 9-21. doi: 10.2201/nonlin. 003.01.002

Baxter, A. J., Scott, K. M., Ferrari, A. J., Norman, R. E., Vos, T., and Whiteford, H. A. (2014). Challenging the myth of an "epidemic" of common mental disorders: trends in the global prevalence of anxiety and depression between 1990 and 2010. Depress. Anxiety 31, 506-516. doi: 10.1002/da. 22230

Beier, E. (1994). [A comparative evaluation of the antidepressive activity of different $\beta$-adrenoblockaders]. Eksp. Klin. Farmakol. 57, 20-21.

Bonde, J. P., Utzon-Frank, N., Bertelsen, M., Borritz, M., Eller, N. H., Nordentoft, M., et al. (2016). Risk of depressive disorder following disasters and military deployment: systematic review with meta-analysis. Br. J. Psychiatry 208, 330-336. doi: 10.1192/bjp.bp.114.157859

Bourin, M., Colombel, M.-C., Malinge, M., and Bradwejn, J. (1991). Clonidine as a sensitizing agent in the forced swimming test for revealing antidepressant activity. J. Psychiatry Neurosci. 16, 199-203.

Bourin, M., Hascoët, M., Colombel, M., Coutts, R. T., and Baker, G. B. (2002). Clonidine potentiates the effects of tranylcypromine, phenelzine and two analogues in the forced swimming test in mice. J. Psychiatry Neurosci. 27, $178-185$.

Bourin, M., Hascoet, M., Colombel, M.-C., Redrobe, J. P., and Baker, G. B. (1996). Differential effects of clonidine, lithium and quinine in the forced swimming test in mice for antidepressants: possible roles of serotoninergic systems. Eur. Neuropsychopharmacol. 6, 231-236. doi: 10.1016/0924-977x(96) 00025-9
Cervo, L., Ross, C., and Samanin, R. (1992). The role of serotonin and dopamine in brain in the antidepressant-like effect of clonidine in the forced swimming test. Neuropharmacology 3, 331-335. doi: 10.1016/0028-3908(92)90064-v

Cervo, L., and Samanin, R. (1991). Clonidine causes antidepressant-like effects in rats by activating a2-adrenoceptors outside the locus coeruleus. Eur. J. Pharmacol. 193, 309-313. doi: 10.1016/0014-2999(91)90144-f

Chen, Y., Bennett, D., Clarke, R., Guo, Y., Yu, C., Bian, Z., et al. (2017). Patterns and correlates of major depression in Chinese adults: a crosssectional study of 0.5 million men and women. Psychol. Med. 47, 958-970. doi: 10.1017/S0033291716002889

Chen, X., Zhao, C., Li, X., Wang, T., Li, Y., Cao, C., et al. (2015). Terazosin activates Pgk1 and Hsp90 to promote stress resistance. Nat. Chem. Biol. 11, 19-25. doi: 10.1038/nchembio.1657

Chopra, K., Kunchandy, J., and Kulkarni, S. K. (1988). Benzodiazepine inverse agonist FG-7142-induced delayed behavioral depression in mice. Arch. Int. Pharmacodyn. Ther. 294, 56-63.

Claustre, Y., Leonetti, M., Santucci, V., Bougault, I., Desvignes, C., Rouquier, L., et al. (2008). Effects of the $\beta 3$-adrenoceptor (Adrb3) agonist SR58611A (amibegron) on serotonergic and noradrenergic transmission in the rodent: relevance to its antidepressant/anxiolytic-like profile. Neuroscience 156, 353-364. doi: 10.1016/j.neuroscience.2008.07.011

Consoli, D., Leggio, G. M., Mazzola, C., Micale, V., and Drago, F. (2007). Behavioral effects of the $\beta 3$ adrenoceptor agonist SR58611A: is it the putative prototype of a new class of antidepressant/anxiolytic drugs? Eur. J. Pharmacol. 573, 139-147. doi: 10.1016/j.ejphar.2007.06.048

Coppen, A. (1967). The biochemistry of affective disorders. Br. J. Psychiatry 113 1237-1264. doi: 10.1192/bjp.113.504.1237

Danysz, W., Kostowski, W., Kozak, W., and Hauptmann, M. (1986). On the role of noradrenergic neurotransmission in the action of desipramine and amitriptyline in animal models of depression. Pol. J. Pharmacol. Pharm. 38, 285-298.

Demin, K. A., Sysoev, M., Chernysh, M. V., Savva, A. K., Koshiba, M., WapplerGuzzetta, E. A., et al. (2019). Animal models of major depressive disorder and the implications for drug discovery and development. Expert Opin. Drug Discov. 14, 365-378. doi: 10.1080/17460441.2019.1575360

Detke, M. J., Wieland, S., and Lucki, I. (1995). Blockade of the antidepressant-like effects of $8.0 \mathrm{H}$-DPAT, buspirone and desipramine in the rat forced swim test by 5HTIA receptor antagonists. Psychopharmacology 119, 47-54. doi: 10.1007/BF02246053

Diehl, D. J., and Gershon, S. (1992). The role of dopamine in mood disorders. Compr. Psychiatry 33, 115-120. doi: 10.1016/0010-440x(92)90007-d

Ding, M., Chen, Y., Luan, H., Zhang, X., Zhao, Z., and Wu, Y. (2019). Dexmedetomidine reduces inflammation in traumatic brain injury by regulating the inflammatory responses of macrophages and splenocytes. Exp. Ther. Med. 18, 2323-2331. doi: 10.3892/etm.2019.7790

Doze, V. A., Handel, E. M., Jensen, K. A., Darsie, B., Luger, E. J., Haselton, J. R., et al. (2009). $\alpha 1 \mathrm{~A}$ - and $\alpha 1 \mathrm{~B}$-adrenergic receptors differentially modulate antidepressant-like behavior in the mouse. Brain Res. 1285, 148-157. doi: 10.1016/j.brainres.2009.06.035

Dremencov, E., El Mansari, M., and Blier, P. (2007a). Distinct electrophysiological effects of paliperidone and risperidone on the firing activity of rat serotonin and norepinephrine neurons. Psychopharmacology 194, 63-72. doi: $10.1007 /$ s00213-007-0818-8

Dremencov, E., El Mansari, M., and Blier, P. (2007b). Noradrenergic augmentation of escitalopram response by risperidone: electrophysiologic studies in the rat brain. Biol. Psychiatry 61, 671-678. doi: 10.1016/j.biopsych.2006.05.015

Duncan, G., Knapp, D., Johnson, K., and Breese, G. (1996). Functional classification of antidepressants based on antagonism of swim stress-induced fos-like immunoreactivity. J. Pharmacol. Exp. Ther. 277, 1076-1089.

Ebada, M. E. (2017). Drug repurposing may generate novel approaches to treating depression. J. Pharm. Pharmacol. 69, 1428-1436. doi: 10.1111/jphp.12815

Emrich, H. M., von Zerssen, D., Möller, H. J., Kissling, W., Cording, C., Schietsch, H. J., et al. (1979). Action of propranolol in mania: comparison of effects of the $\mathrm{d}$ - and the 1-stereoisomer. Pharmakopsychiatr. Neuropsychopharmakol. 12, 295-304. doi: 10.1055/s-0028-1094624

Evangelista, S., Borsini, F., and Meli, A. (1987). Evidence that muscimol acts in the forced swimming test by activating the rat dopaminergic system. Life Sci. 41 , 2679-2684. doi: 10.1016/0024-3205(87)90283-9 
Ferrari, F., Cassinadri, M., Tartoni, P. L., and Tampieri, A. (1991). Effects of B-HT 920 in the tail-suspension test. Pharmacol. Res. 24, 75-81. doi: 10.1016/10436618(91)90067-8

Finnegan, K., Terwillger, M. M., Berger, P. A., Holhster, L. E., and Csernansky, J. G. (1987). A comparison of the neurochemical and behavioral effects of clenbuterol and desipramine.. Eur. J. Pharmacol. 134, 131-136. doi: 10.1016/0014-2999(87)90158-0

Fitzgerald, P. J., and Watson, B. O. (2019). in vivo electrophysiological recordings of the effects of antidepressant drugs. Exp. Brain Res. 237, 1593-1614. doi: 10.1007/s00221-019-05556-5

Giustino, T. F., Fitzgerald, P. J., and Maren, S. (2016). Revisiting propranolol and PTSD: memory erasure or extinction enhancement? Neurobiol. Learn. Mem. 130, 26-33. doi: 10.1016/j.nlm.2016.01.009

Giustino, T. F., and Maren, S. (2018). Noradrenergic modulation of fear conditioning and extinction. Front. Behav. Neurosci. 12:43. doi: 10.3389/fnbeh. 2018.00043

Gresch, P. J., Sved, F., Zigroond, J., and Finlay, J. M. (1995). Local influence of endogenous norepinephrine on extracellular dopamine in rat medial prefrontal cortex. J. Neurochem. 65, 111-116. doi: 10.1046/j.1471-4159.1995.65010111.x

Groft, M. L., Normann, M. C., Nicklas, P. R., Jagielo-Miller, J. E., and McLaughlin, P. J. (2019). Biphasic effects of 5-HT1A agonism on impulsive responding are dissociable from effects on anxiety in the variable consecutive number task. Naunyn Schmiedebergs Arch. Pharmacol. 392, 1455-1464. doi: 10.1007/s00210-019-01684-5

Gu, L., Liu, Y.-J., Wang, Y.-B., and Yi, L. T. (2012). Role for monoaminergic systems in the antidepressant-like effect of ethanol extracts from Hemerocallis citrina. J. Ethnopharmacol. 139, 780-787. doi: 10.1016/j.jep.2011.11.059

Guiard, B. P., El Mansari, M., Merali, Z., and Blier, P. (2008). Functional interactions between dopamine, serotonin and norepinephrine neurons: an in-vivo electrophysiological study in rats with monoaminergic lesions. Int. J. Neuropsychopharmacol. 11, 625-639. doi: 10.1017/S1461145707008383

Guo, K., Ma, Q., Li, J., Wang, Z., Shan, T., Li, W., et al. (2013). Interaction of the sympathetic nerve with pancreatic cancer cells promotes perineural invasion through the activation of STAT3 signaling. Mol. Cancer Ther. 12, 264-273. doi: 10.1158/1535-7163.MCT-12-0809

Hardy, M.-C., Lecrubier, Y., and Widlocher, D. (1986). Efficacy of clonidine in 24 patients with acute mania. Am. J. Psychiatry 143, 1450-1453. doi: 10.1176/ajp.143.11.1450

Hascoät, M., Bourin, M., and Bradwejn, J. (1991). Behavioral models in mice. implication of the $\alpha$ noradrenergic system. Prog. Neuropsychopharmacol. Biol. Psychiatry 15, 825-840. doi: 10.1016/0278-5846(91)90011-o

Hascoet, M., Bourin, M., and Khimake, S. (1994). Additive effect of lithium and clonidine with 5-HT1A agonists in the forced swimming test. Prog. Neuropsychopharmacol. Biol. Psychiatry 18, 381-396. doi: 10.1016/02785846(94)90070-1

Hasin, D. S., Sarvet, A. L., Meyers, J. L., Saha, T. D., Ruan, W. J., Stohl, M., et al. (2018). Epidemiology of adult DSM-5 major depressive disorder and its specifiers in the United States. JAMA Psychiatry 75, 336-346. doi: 10.1001/jamapsychiatry.2017.4602

Hosang, G. M., Shiles, C., Tansey, K. E., McGuffin, P., and Uher, R. (2014). Interaction between stress and the BDNF Val66Met polymorphism in depression: a systematic review and meta-analysis. BMC Med. 12:7. doi: 10.1186/1741-7015-12-7

Ingram, W. M., Baker, A. M., Bauer, C. R., Brown, J. P., Goes, F. S., Larson, S., et al. (2020). Defining major depressive disorder cohorts using the EHR: multiple phenotypes based on ICD-9 codes and medication orders. Neurol. Psychiatry Brain Res. 36, 18-26. doi: 10.1016/j.npbr.2020.02.002

Janowsky, D. S., El-Yousef, M. K., Davis, J. M., and Sekerke, H. J. (1972). A cholinergic-adrenergic hypothesis of mania and depression. Lancet 2, 632-635. doi: 10.1016/s0140-6736(72)93021-8

Kaster, M. P., Raupp, I., Binfaré, R. W., Andreatini, R., and Rodrigues, A. L. S. (2007). Antidepressant-like effect of lamotrigine in the mouse forced swimming test: evidence for the involvement of the noradrenergic system. Eur. J. Pharmacol. 565, 119-124. doi: 10.1016/j.ejphar.2007.03.003

Kessing, L. V., Rytgaard, H. C., Ekstrøm, C. T., Torp-Pedersen, C., Berk, M., and Gerds, T. A. (2020). Antihypertensive drugs and risk of depression: a nationwide population-based study. Hypertension 76, 1263-1279. doi: 10.1161/HYPERTENSIONAHA.120.15605
Kim, C., Duan, L., Phan, D. Q., and Lee, M. S. (2019). Frequency of utilization of $\beta$ blockers in patients with heart failure and depression and their effect on mortality. Am. J. Cardiol. 124, 746-750. doi: 10.1016/j.amjcard.2019. 05.054

Kitada, Y., Miyauchi, T., Kosasa, T., and Satoh, S. (1983). Further studies on the suppressing effect of isoproterenol on the immobility-reducing action of desipramine in the forced swimming test. Jpn. J. Pharmacol. 33, 867-873. doi: $10.1254 /$ jjp. 33.867

Koella, W. P. (1985). CNS-related (side-)effects of blockers with special reference to mechanisms of action. Eur. J. Clin. Pharmacol. 28, 55-63. doi: 10.1007/BF00543711

Kotagale, N. R., Tripathi, S. J., Aglawe, M. M., Chopde, C. T., Umekar, M. J., and Taksande, B. G. (2013). Evidences for the agmatine involvement in antidepressant like effect of bupropion in mouse forced swim test. Pharmacol. Biochem. Behav. 107, 42-47. doi: 10.1016/j.pbb.2013.03.019

Kurosawa, N., Shimizu, K., and Seki, K. (2016). The development of depression-like behavior is consolidated by IL-6-induced activation of locus coeruleus neurons and IL-1 $\beta$-induced elevated leptin levels in mice. Psychopharmacology 233, 1725-1737. doi: 10.1007/s00213-0154084-x

Liu, X., Peprah, D., and Gershenfeld, H. K. (2003). Tail-suspension induced hyperthermia: a new measure of stress reactivity. J. Psychiatr. Res. 37, 249-259. doi: 10.1016/s0022-3956(03)00004-9

Maity, S., Chandanathil, M., Millis, R. M., and Connor, S. A. (2020). Norepinephrine stabilizes translation-dependent, homosynaptic long-term potentiation through mechanisms requiring the cAMP sensor Epac, mTOR and MAPK. Eur. J. Neurosci. 52, 3679-3688. doi: 10.1111/ejn. 14735

Malikowska, N., Fijakowski, €., Nowaczyk, A., Popik, P., and Saat, K. (2017). Antidepressant-like activity of venlafaxine and clonidine in mice exposed to single prolonged stress-a model of post-traumatic stress disorder. Pharmacodynamic and molecular docking studies. Brain Res. 1673, 1-10. doi: 10.1016/j.brainres.2017.08.001

Malinge, M., Bourin, M., Colombel, M. C., and Larousse, C. (1988). Additive effects of clonidine and antidepressant drugs in the mouse forced-swimming test. Psychopharmacology 96, 104-109. doi: 10.1007/BF024 31541

Malinge, M., Colombel, M., and Bourin, M. (1989). Mechanism of action of clonidine in the forced-swimming test in mice. Encephale 15, 37-41.

Masuda, Y., Ohnuma, S., and Sugiyama, T. (2001). $\alpha$ 2-adrenoceptor activity induces the antidepressant-like glycolipid in mouse forced swimming. Methods Find. Exp. Clin. Pharmacol. 23, 19-21. doi: 10.1358/mf.2001.23.1. 619175

Miller, A. H., Maletic, V., and Raison, C. L. (2009). Inflammation and its discontents: the role of cytokines in the pathophysiology of major depression. Biol. Psychiatry 65, 732-741. doi: 10.1016/j.biopsych.2008.11.029

Mineur, Y. S., Bentham, M. P., Zhou, W.-L., Plantenga, M. E., McKee, S. A., and Picciotto, M. R. (2015). Antidepressant-like effects of guanfacine and sex-specific differences in effects on c-fos immunoreactivity and pairedpulse ratio in male and female mice. Psychopharmacology 232, 3539-3549. doi: 10.1007/s00213-015-4001-3

Mineur, Y. S., Cahuzac, E. L., Mose, T. N., Bentham, M. P., Plantenga, M. E., Thompson, D. C., et al. (2018). Interaction between noradrenergic and cholinergic signaling in amygdala regulates anxiety- and depressionrelated behaviors in mice. Neuropsychopharmacology 43, 2118-2125. doi: 10.1038/s41386-018-0024-x

Mitchell, P. B. (2004). Therapeutic drug monitoring of non-tricyclic antidepressant drugs. Clin. Chem. Lab. Med. 42, 1212-1218. doi: 10.1515/CCLM.2004.243

Muguruza, C., Rodríguez, F., Rozas, I., Meana, J. J., Urigüen, L., and Callado, L. F. (2013). Antidepressant-like properties of three new $\alpha 2$ adrenoceptor antagonists. Neuropharmacology 65, 13-19. doi: 10.1016/j. neuropharm.2012.09.003

Muthalif, M. M., Benter, I. F., Karzoun, N., Fatima, S., Harper, J., Uddin, M. R., et al. (1998). 20-Hydroxyeicosatetraenoic acid mediates calcium/calmodulindependent protein kinase II-induced mitogen-activated protein kinase activation in vascular smooth muscle cells. Proc. Natl. Acad. Sci. U S A 95, 12701-12706. doi: 10.1073/pnas.95.21.12701 
Nemeth, D. G., and Mckenzie Chustz, K. (2020). Treating comorbid childhood bipolar disorder and ADHD. CNS Spectr. 25, 283-284. doi: 10.1017/S1092852920000486

O’Neill, M. F., Osborne, D. J., Woodhouse, S. M., and Conway, M. W. (2001). Selective imidazoline I2 ligands do not show antidepressant-like activity in the forced swim test in mice. J. Psychopharmacol. 15, 18-22. doi: 10.1177/026988110101500104

Overstreet, D. H., Stemmelin, J., and Griebel, G. (2008). Confirmation of antidepressant potential of the selective $\beta 3$ adrenoceptor agonist amibegron in an animal model of depression. Pharmacol. Biochem. Behav. 89, 623-626. doi: 10.1016/j.pbb.2008.02.020

Parale, M. P., Chakravarti, S., and Kulkarni, S. K. (1987). Evidence of $\beta$ adrenergic involvement in forced swimming-induced behavioural despair of mice. Methods Find. Exp. Clin. Pharmacol. 9, 35-38.

Parale, M. P., and Kulkarni, S. K. (1986). Clonidine-induced behavioural despair in mice: reversal by antidepressants. Psychopharmacology 89, 171-174. doi: 10.1007/BF00310623

Park, M. J., Yoo, S. W., Choe, B. S., Dantzer, R., and Freund, G. G. (2012). Acute hypoglycemia causes depressive-like behaviors in mice. Metab. Clin. Exp. 61, 229-236. doi: 10.1016/j.metabol.2011.06.013

Pesarico, A. P., Sampaio, T. B., Stangherlin, E. C., Mantovani, A. C., Zeni, G., and Nogueira, C. W. (2014). The antidepressant-like effect of 7-fluoro-1,3diphenylisoquinoline-1-amine in the mouse forced swimming test is mediated by serotonergic and dopaminergic systems. Prog. Neuropsychopharmacol. Biol. Psychiatry 54, 179-186. doi: 10.1016/j.pnpbp.2014.06.001

Polis, A. J., Fitzgerald, P. J., Hale, P. J., and Watson, B. O. (2019). Rodent ketamine depression-related research: finding patterns in a literature of variability. Behav. Brain Res. 376:112153. doi: 10.1016/j.bbr.2019.112153

Poncelet, M., Martin, P., Danti, S., Simon, P., and Soubrié, P. (1987). Noradrenergic rather than GABAergic processes as the common mediation of the antidepressant profde of GABA agonists and imipramine-like drugs in animals. Pharmacol. Biochem. Behav. 28, 321-326. doi: 10.1016/00913057(87)90447-3

Redrobe, J. P., and Bourin, M. (1997). Effects of pretreatment with clonidine, lithium and quinine on the activities of antidepressant drugs in the mouse tail suspension test. Fundam. Clin. Pharmacol. 11, 381-386. doi: 10.1111/j.14728206.1997.tb00199.x

Redrobe, J. P., and Bourin, M. (1998). Clonidine potentiates the effects of 5-HT, 5-HT and 5-HT 1A 1B 2A/2C antagonists and 8-OH-DPAT in the mouse forced swimming test. Eur. Neuropsychopharmacol. 8, 169-173. doi: 10.1016/s0924-977x(97)00073-4

Rénéric, J.-P., Bouvard, M., and Stinus, L. (2002). In the rat forced swimming test, chronic but not subacute administration of dual 5-HT/NA antidepressant treatments may produce greater effects than selective drugs. Behav. Brain Res. 136, 521-532. doi: 10.1016/s0166-4328(02)00203-6

Ribeiro, C. A. S., and Pupo, A. S. (2015). Involvement of $\alpha 1 \mathrm{~B}$-adrenoceptors in the anti-immobility effect of imipramine in the tail suspension test. Eur. J. Pharmacol. 750, 39-42. doi: 10.1016/j.ejphar.2015.01.010

Rosen, R., and Kostis, J. (1985). Biobehavioral sequellae associated with adrenergic-inhibiting antihypertensive agents: a critical review. Health Psychol. 4, 579-604. doi: 10.1037//0278-6133.4.6.579

Ruhé, H. G., Huyser, J., Swinkels, J. A., and Schene, A. H. (2006). Switching antidepressants after a first selective serotonin reuptake inhibitor in major depressive disorder: a systematic review. J. Clin. Psychiatry 67, 1836-1855. doi: $10.4088 /$ jcp.v67n1203

Sallinen, J., Haapalinna, A., MacDonald, E., Viitamaa, T., LähdesmäKi, J., Rybnikova, E., et al. (1999). Genetic alteration of the 2-adrenoceptor subtype $c$ in mice affects the development of behavioral despair and stressinduced increases in plasma corticosterone levels. Mol. Psychiatry 4, 443-452. doi: 10.1038/sj.mp.4000543

Schildkbaut, J. J. (1965). The catecholamine hypothesis of affective disorders: a review of supporting evidence. Am. J. Psychiatry 122, 509-522. doi: 10.1176/ajp. 122.5.509

Schmaal, L., Hibar, D. P., Sämann, P. G., Hall, G. B., Baune, B. T., Jahanshad, N., et al. (2017). Cortical abnormalities in adults and adolescents with major depression based on brain scans from 20 cohorts worldwide in the ENIGMA major depressive disorder working group. Mol. Psychiatry 22, 900-909. doi: $10.1038 / \mathrm{mp} .2016 .60$
Schreiber, R., and De Vry, J. (1993). Neuroanatomical basis for the antidepressant-like effects of the 5-HT(1A) receptor agonists 8-OH-DPAT and ipsapirone in the rat forced swimming test. Behav. Pharmacol. 4, 625-636. doi: 10.1097/00008877-199312000-00008

Sekio, M., and Seki, K. (2015). Lipopolysaccharide-induced depressive-like behavior is associated with $\alpha 1$-adrenoceptor dependent downregulation of the membrane GluR1 subunit in the mouse medial prefrontal cortex and ventral tegmental area. Int. J. Neuropsychopharmacol. 18:pyu005. doi: 10.1093/ijnp/pyu005

Skrebuhhova, T., Allikmets, L., and Matto, V. (1999). Effects of anxiogenic drugs in rat forced swimming test. Methods Find. Exp. Clin. Pharmacol. 21, 173-178. doi: 10.1358/mf.1999.21.3.534826

Stemmelin, J., Cohen, C., Terranova, J.-P., Lopez-Grancha, M., Pichat, P., Bergis, O., et al. (2008). Stimulation of the $\beta 3$-adrenoceptor as a novel treatment strategy for anxiety and depressive disorders. Neuropsychopharmacology 33, 574-587. doi: 10.1038/sj.npp.1301424

Stemmelin, J., Cohen, C., Yalcin, I., Keane, P., and Griebel, G. (2010). Implication of $\beta 3$-adrenoceptors in the antidepressant-like effects of amibegron using Adrb3 knockout mice in the chronic mild stress. Behav. Brain Res. 206, 310-312. doi: 10.1016/j.bbr.2009.09.003

Stone, E. A., Lin, Y., Sarfraz, Y., and Quartermain, D. (2011). Antidepressantlike action of intracerebral 6-fluoronorepinephrine, a selective full $\alpha$-adrenoceptor agonist. Int. J. Neuropsychopharmacol. 14, 319-331. doi: $10.1017 /$ S1461145710000507

Stone, E. A., and Quartermain, D. (1999). $\alpha$-1-noradrenergic neurotransmission, corticosterone, and behavioral depression. Biol. Psychiatry 46, 1287-1300. doi: 10.1016/s0006-3223(99)00234-6

Sugimoto, Y., Yamamoto, M., Tagawa, N., Kobayashi, Y., Mitsui-Saitoh, K., Hotta, Y., et al. (2011). Differences between mice strains in response to paroxetine in the forced swimming test: involvement of serotonergic or noradrenergic systems. Eur. J. Pharmacol. 672, 121-125. doi: 10.1016/j. parkreldis.2021.08.019

Taksande, B. G., Kotagale, N. R., Tripathi, S. J., Ugale, R. R., and Chopde, C. T. (2009). Antidepressant like effect of selective serotonin reuptake inhibitors involve modulation of imidazoline receptors by agmatine. Neuropharmacology 57, 415-424. doi: 10.1016/j.neuropharm.2009.06.035

Tamburella, A., Micale, V., Leggio, G. M., and Drago, F. (2010). The $\beta 3$ adrenoceptor agonist, amibegron (SR58611A) counteracts stress-induced behavioral and neurochemical changes. Eur. Neuropsychopharmacol. 20, 704-713. doi: 10.1016/j.euroneuro.2010.04.006

Tanis, K. Q., and Duman, R. S. (2007). Intracellular signaling pathways pave roads to recovery for mood disorders. Ann. Med. 39, 531-544.doi: 10.1080/07853890701483270

Teste, J., Martin, I., and Rinjard, P. (1990). Electrotherapy in mice: dopaminergic and noradrenergic effects in the Tail Suspension Test. Fund. Clin. Pharmacol. 4, 39-47. doi: 10.1111/j.1472-8206.1990.tb01015.x

Ulrich, S., Ricken, R., Buspavanich, P., Schlattmann, P., and Adli, M. (2020). Efficacy and adverse effects of tranylcypromine and tricyclic antidepressants in the treatment of depression: a systematic review and comprehensive meta-analysis. J. Clin. Psychopharmacol. 40, 63-74. doi: 10.1097/JCP. 0000000000001153

Uys, M. M., Shahid, M., Sallinen, J., and Harvey, B. H. (2017). The $\alpha 2$ Cadrenoceptor antagonist, ORM-10921, exerts antidepressant-like effects in the Flinders Sensitive Line rat. Behav. Pharmacol. 28, 9-18. doi: 10.1097/FBP. 0000000000000261

Vaidya, V. A., and Duman, R. S. (2001). Depression-emerging insights from neurobiology. Br. Med. Bull. 57, 61-79. doi: 10.1093/bmb/ 57.1 .61

Van Gaalen, M., Kawahara, H., Kawahara, Y., and Westerink, B. (1997). The locus coeruleus noradrenergic system in the rat brain studied by dual-probe microdialysis. Brain Res. 763, 56-62. doi: 10.1016/s0006-8993(97) 00416-2

Vijayraghavan, S., Wang, M., Birnbaum, S. G., Williams, G. V., and Arnsten, A. F. T. (2007). Inverted-U dopamine D1 receptor actions on prefrontal neurons engaged in working memory. Nat. Neurosci. 10, 376-384. doi: $10.1038 / \mathrm{nn} 1846$

Waldmeier, P. C. (1981). Noradrenergic transmission in depression: under-or overfunction? Pharmakopsychiatria 14, 3-9. doi: 10.1055/s-2007-1019557 
Wohleb, E. S., Franklin, T., Iwata, M., and Duman, R. S. (2016). Integrating neuroimmune systems in the neurobiology of depression. Nat. Rev. Neurosci. 17, 497-511. doi: 10.1038/nrn.2016.69

Wu, Z. H., Zhang, Q. J., Du, C. X., Xi, Y., Li, W. J., Guo, F. Y., et al. (2017). Prelimbic $\alpha 1$-adrenoceptors are involved in the regulation of depressive-like behaviors in the hemiparkinsonian rats. Brain Res. Bull. 134, 99-108. doi: 10.1016/j.brainresbull.2017. 07.011

Yanagawa, Y., Matsumoto, M., and Togashi, H. (2010). Enhanced dendritic cell antigen uptake via $\alpha 2$ adrenoceptor-mediated PI3K activation following brief exposure to noradrenaline. J. Immunol. 185, 5762-5768. doi: 10.4049/jimmunol.1001899

Zaidi, S., Atrooz, F., Valdez, D., Liu, H., Kochi, C., Bond, R. A., et al. (2020). Protective effect of propranolol and nadolol on social defeat-induced behavioral impairments in rats. Neurosci. Lett. 725:134892. doi: 10.1016/j. neulet.2020.134892

Zeidan, M. P., Zomkowski, A. D. E., Rosa, A. O., Rodrigues, A. L. S., and Gabilan, N. H. (2007). Evidence for imidazoline receptors involvement in the agmatine antidepressant-like effect in the forced swimming test. Eur. J. Pharmacol. 565, 125-131. doi: 10.1016/j.ejphar.2007. 03.027
Zhang, H.-T., Whisler, L. R., Huang, Y., Xiang, Y., and O’Donnell, J. M. (2009). Postsynaptic $\alpha-2$ adrenergic receptors are critical for the antidepressant-like effects of desipramine on behavior. Neuropsychopharmacology 34, 1067-1077. doi: 10.1038/npp.2008.184

Conflict of Interest: The author declares that the research was conducted in the absence of any commercial or financial relationships that could be construed as a potential conflict of interest.

Publisher's Note: All claims expressed in this article are solely those of the authors and do not necessarily represent those of their affiliated organizations, or those of the publisher, the editors and the reviewers. Any product that may be evaluated in this article, or claim that may be made by its manufacturer, is not guaranteed or endorsed by the publisher.

Copyright (C) 2021 Fitzgerald. This is an open-access article distributed under the terms of the Creative Commons Attribution License (CC BY). The use, distribution or reproduction in other forums is permitted, provided the original author(s) and the copyright owner(s) are credited and that the original publication in this journal is cited, in accordance with accepted academic practice. No use, distribution or reproduction is permitted which does not comply with these terms. 